Original Article

\title{
Preventive Effect and Mechanism of Ethyl Acetate Extract of Sceptridium ternatum in Monocrotaline-Induced Pulmonary Arterial Hypertension*
}

\author{
XIN Wen-xiu , LI Qing-lin, FANG Luo, ZHONG Li-ke, ZHENG Xiao-wei , and HUANG Ping
}

\begin{abstract}
Objective: To observe the effect and molecular mechanism of ethyl acetate extract of Sceptridium ternatum (STE) on the monocrotaline (MCT)-induced pulmonary arterial hypertension (PAH). Methods: The main chemical components of Sceptridium ternatum were determined, and the effects in PAH rats were observed. A total of 140 Sprague Dawley rats were randomly and equally divided into the normal group, the model group, the Bosentan group, and the STE groups $(2.5,5,10 \mathrm{~g} / \mathrm{kg})$ by the random number table method. The characteristic indicators of PAH were measured, and immunohistochemistry was used to observe the lung tissue of rats. Morphological changes of the lung tissue were observed under the light microscope. Results: Compared with the normal group, rats in the model group showed a significant increase in right ventricular free wall thickness (RVFWT), mean pulmonary arterial pressure (mPAP), mean right ventricular pressure (mRVP), max right ventricular pressure (max RVP), weight of right ventricle (RV), and lung index (LI), while a significant decrease in pulmonary artery acceleration time (PAAT, $P<0.01$ ). Compared with the model group, rats treated with STE had a significant decrease of RVFWT, mPAP, mRVP, max RVP, and RV, while a significant increase of PAAT $(P<0.01)$. After injection of MCT, nuclear factor- $\kappa$ B (NF- $\kappa$ B) p65 and $\alpha$-smooth muscle actin ( $\alpha$-SMA) expression levels were up-regulated, and on the contrary, the treatment groups showed a significant downregulation without dose-dependent trend. Conclusions: STE can relieve the PAH in rats. STE may relieve pulmonary vascular disease and pulmonary injury by down-regulating the expression of NF- $\kappa$ B p65 and $\alpha$-SMA. KEYWORDS pulmonary arterial hypertension, ethyl acetate extract of Sceptridium ternatum, mechanism, Chinese medicine
\end{abstract}

Pulmonary heart disease is caused by lung tissue, pulmonary vascular or thoracic chronic lesions and functional changes, resulting in pulmonary vascular resistance enhanced, pulmonary artery pressure increased, right ventricular hypertrophy dilated, and a class of diseases with right heart failure. ${ }^{(1)}$ In addition, it is also accompanied by a number of organizational dysfunction and endogenous changes in the molecular level, ${ }^{(2)}$ with high prevalence, high mortality and high cost of the characteristics of a serious threat to human health. Pulmonary arterial hypertension (PAH) is the pathophysiological basis of the occurrence and development of pulmonary heart disease. Continuous $\mathrm{PAH}$ will eventually lead to right ventricular hypertrophy and enlargement, right heart failure, higher pulmonary artery pressure, or even worse prognosis. ${ }^{(3)}$

The main pathological changes of pulmonary heart disease are $\mathrm{PAH}$ and right heart hypertrophy, and $\mathrm{PAH}$ is the central link in the pathogenesis of pulmonary heart disease. The main treatment of $\mathrm{PAH}$ aims to improve respiratory function, correct hypoxia and carbon dioxide retention, anticoagulant, vasodilator agent application. ${ }^{(4)}$ However, mature drugs and measures are relatively deficient. In recent years, Chinese medicine has made some progress in the prevention and

(C)The Chinese Journal of Integrated Traditional and Western Medicine Press and Springer-Verlag GmbH Germany, part of Springer Nature 2018

*Supported by the Zhejiang Province Traditional Chinese Medicine Key Scientific Research Fund Project (No. 2016ZZ009); Zhejiang Province Traditional Chinese Medicine Scientific Research Fund Project (No. 2017ZB022); Zhejiang Province Traditional Chinese Medicine Youth Scientific Research Fund Project (No. 2011ZQ001); Zhejiang Medicine and Scientific Research Fund Project (No. 2015KYB061); Zhejiang Province Health Innovation Personnel Training Program of Huang Ping (No. 201663); and The Second Level Training Program of Zhejiang Province's 151 Talents Project of Huang Ping (No. 2016118) Department of Pharmacy, Zhejiang Cancer Hospital, Hangzhou (310022), China

Correspondence to: Dr. HUANG Ping, Tel: 86-571-88122201, E-mail: huangping1841@zjcc.org.cn

DOI: https://doi.org/10.1007/s11655-018-2573-6 
treatment of pulmonary heart disease. ${ }^{(5)}$ Scepteridium ternatum has the functions of anti-inflammation, antiallergy, anti-oxidation and anti-proliferation. ${ }^{(6)}$ Scepteridium ternatum can enhance reticuloendothelial system function in mice and improve the immune function of mice. ${ }^{(7)}$ Wang, et $\mathrm{al}^{(8)}$ reported that, with strong preventive effect on cancer chemically, Scepteridium ternatum showed its function of improving immunity.

Scepteridium ternatum is a commonly used herb in southern China, with the function of stopping cough. Chinese people often use it for the treatment of bronchial asthma, whooping cough, and children's febrile convulsive twitch disease. In this study, the rat model of pulmonary heart disease induced by monocrotaline (MCT) was established to observe preventive effect of ethyl acetate extract of Scepteridium ternatum (STE) on PAH.

\section{METHODS}

\section{Animals}

Totally 140 healthy adult Sprague Dawley (SD) rats, half male and half female weighing $(200 \pm 20) \mathrm{g}$, were provided by Shanghai Kay Laboratory Animal Co., Ltd. [animal production license No. SCXK (Shanghai) 2008-0016, animal qualified certificate No. 2008001630484]. All animal protocols were approved by the Animal Care and Use Committee at Zhejiang University. Male and female rats were maintained separately with free access to food and water under the temperature of $20-26{ }^{\circ} \mathrm{C}$, the humidity of $40 \%-70 \%$, and a 12-h light-dark cycle.

\section{Medicines and Reagents}

Scepteridium ternatum was collected from Lishui, Zhejiang province. Voucher specimens (No. ZJ-20120926) were carefully identified by Prof. CHEN Xi-lin from Zhejiang Chinese Medical University, and they met the relevant requirements of STE in the Chinese Pharmacopoeia.

Bosentan was purchased from Allpack Group Middlemore Lane West, United Kingdom. Normal saline was purchased from Hangzhou Minsheng Pharmaceutical Co., Ltd., China (batch No. 21205062). MCT was purchased from Shanghai biochempartner Co., Ltd., China (batch No. 12120505).

\section{Preparation and Identification of Scepteridium Ternatum \\ Scepteridium ternatum herb weighing $15 \mathrm{~kg}$, was added with 10 times volume of $70 \%$ ethanol for}

heat reflux extraction. Extraction was performed for 3 times, $2 \mathrm{~h}$ each time. The filtrates were combined and concentrated under reduced pressure to obtain the extract. After dissolution with water, equal volume of petroleum ether, chloroform, ethyl acetate and n-butanol were added successively, for repeated extraction. Finally, $231 \mathrm{~g}$ petroleum ether extract (1.53\%), $86.7 \mathrm{~g}$ chloroform extract $(0.57 \%), 70 \mathrm{~g}$ ethyl acetate extract $(0.46 \%)$ and $293.5 \mathrm{~g} \mathrm{n}$-butanol extract (1.94\%) were obtained.

\section{Analysis of Extracts from Scepteridium Ternatum}

All analysis of STE was performed by using high performance liquid chromatography tandem mass spectrometry (HPLC-MS/MS) methods. Chromatographic separations were achieved by using a Waters Acquity BEHC18 column $(2.1 \mathrm{~mm} \times 150 \mathrm{~mm}$, particle size $1.7 \mu \mathrm{m}$, Waters, Wexford, Ireland). The mobile phase conditions were as follows: A was water with $0.5 \%$ formic acid, and $B$ was acetonitrile. The gradient elution procedures were that $0-10 \mathrm{~min}, A: B=90: 10(\mathrm{v} / \mathrm{v})$; 10-15 min, $A: B$ by $90: 10$ gradually rose to $70: 30(\mathrm{v} / \mathrm{v})$; $15-45 \mathrm{~min}, A: B$ by $70: 30$ gradually rose to $10: 90(\mathrm{v} / \mathrm{v})$ 45-60 min, $A: B=10: 90(v / v)$.

\section{Animal Grouping}

A total of 140 SD rats were randomly divided into 7 groups by the random number table method: the normal control group, the model group, the bosentan group and the STE high-, medium-, and low-dose groups, and there were 20 rats in each group.

\section{Establishment and Administration of the PAH Model}

In addition to the normal control group, the other groups were injected subcutaneously MCT (ethanol:saline $=2: 8$ as solvent) $60 \mathrm{mg} / \mathrm{kg}$ on the neck back, while the normal control group, with subcutaneous injection of the same amount of ethanol: saline $=2: 8$. After the second day of injection, each group began to receive the corresponding dose of the solvent or the drug by way of gavage once daily. The normal control group and the model group were given the normal saline with $10 \mathrm{~mL} / \mathrm{kg}$ dose each time; The positive control group were treated with bosentan with $0.1 \mathrm{~g} / \mathrm{kg}$ dose each time. The STE high-, medium- and low-dose groups were administered $10,5,2.5 \mathrm{~g} / \mathrm{kg}$ STE extract doses respectively. After continuous administration for 21 days, animals were sacrificed for the determination of the corresponding index. All the experimental animals weighed once every other day, drug doses were adjusted according 
to the weight. According to the requirements of the animal ethics committee of Zhejiang University, the animals were killed by cervical dislocations.

\section{General Observation}

Mental state of the rats was observed during the experiment, as well as conditions including eating and drinking, the degree of gloss shiny fur, behavior, urine, and animal body weight was measured once a week.

\section{Evaluation of Indices in Rats by Color Doppler Imaging}

Twenty-one days after the establishment of the model, the right ventricular free wall thickness (RVFWT), pulmonary artery acceleration time (PAAT) and tricuspid regurgitation were measured by color Doppler imaging (LSH10-1A, China) in all surviving animals.

\section{Detection of Right Ventricular Pressure and Pulmonary Artery Pressure}

A special PE50 tube was used to enter the right ventricle from the right atrium of rats, which finally reached the pulmonary artery to record the corresponding pressure and waveform, including mean pulmonary artery pressure (mPAP), mean right ventricular pressure (mRVP) and max right ventricular pressure (max RVP).

\section{Right Heart Hypertrophy Index Calculation}

Hearts were isolated, and the right ventricle was separated carefully from the left ventricle plus septum $(L V+S)$. Then the $L V+S$ was weighed. Ventricles were blotted dry and weighed separately to measure the right ventricle (RV) free wall weight so as to determine the index of right ventricle hypertrophy $(\mathrm{RVHI}) . \mathrm{RVHI}=[\mathrm{RV} /(\mathrm{LV}+\mathrm{S})] \times 100 \%$.

At the end of the experiment, the rats were sacrificed by cervical dislocations, and the lungs and hearts were isolated. Saline was injected into the pulmonary arteries to wash out the blood. Hilars of lungs were cut off and lungs wet weight (wW) were measured. Lung index $(\mathrm{LI})=$ lung $w W /$ body weight $(B W) \times 100 \%$.

\section{Pathological Morphology of Hematoxylin-Eosin Staining in Lung Tissue}

The samples were fixed 48 h with $4 \%$ paraformaldehyde and cut along the hilum. Then, the left lung tissue block was taken. After washing and dehydration, transparent, leaching wax, embedding, cutting, and the dyeing processing, small pulmonary artery was observed by optical microscopy (RWD, 77001, China).

\section{Detection of Immunohistochemical Index}

At the end of the experiment, $6 \mu \mathrm{m}$ frozen sections of rats lungs were prepared and stored at $-20^{\circ} \mathrm{C}$ for immunohistochemistry (IHC) staining. IHC staining was carried out according to the instructions of HSP007/8 immunohistochemical kit to detect the expression of alpha smooth muscle actin ( $\alpha$-SMA) and nuclear factor- $\kappa$ B (NF- $\kappa$ B) p65.

\section{Statistical analysis}

Data were analyzed with SPSS20.0 version software. For analysis of variance between groups, $t$ test was used for comparison. All results were presented as the mean \pm standard deviation $(\bar{x} \pm s)$. $P<0.05$ was considered significant difference.

\section{RESULTS}

\section{Identification of Main Chemical Components of STE}

The structure of the major chemical components were as follows:(1)2-(3,4-Dihydroxy-phenyl)5,7-dihydroxy-chromen-4-on;(2)quercetin-3-Lrhamnoside;(3)kaempferol3-rhamnoside;(4)kaempferol3,7-O-bis(alpha-L-rhamnoside);(5)Kaempferol-3-Oalpha-L-(beta-D-glucopyranosyl)-2-rhamnopyranoside;(6) quercetin3-O-beta-D-glucopyranosyl(1->2)-alpha-Lrhamnopyranosyl-7-O-alpha-L-rhamnopyranoside;(7) kaempferol3-O-alpha-L-(2,3-di-O-beta-Dglucopyranosyl)-alpha-Lrhamnopyranoside. The main chemical compositions of STE are shown by the form of attached materials in Appendix.

\section{Effect of STE on General Conditions of Rats}

During the experiment, rats in the normal control group behaved normally in mental state, food, water intake, fur outlook, action behavior, responsive action and urine amount. The rats in the model group behaved normally in mental state, food, water intake at the early time. But at the third week, the rats became depressed, and food intake decreased with no change of water intake and responsive action, and the fur had luster but shedding when crawled. At the end of the experiment, most rats seemed short of breath, lost body weight with dull fur and curled up with less dynamic. Compared with animals in the model group, animals in the treatment group showed basically free movement and stable respiration, no marked cyanosis 
of lips, more glossy hair and food intake, while loss of body weight was more significant.

Twenty-one days after modeling, compared with the model group, the animal weight of the control group and the STE treatment groups significantly increased $(P<0.01)$, and the results are shown in Table 1. Two rats of the model group died respectively on the 16th and 20th day because of natural causes (autopsy confirmed that the death was caused by heart and lung diseases).

\section{Effect of STE on RVFWT and PAAT in PAH rats}

After 3 weeks of modeling, compared with the normal control group, RVFWT of the model group showed significant increase $(P<0.01)$; compared with the model group, RVFWT of the STE high-, mediumand low-dose groups were significantly decreased $(P<0.01$, Figure 1). Meanwhile, compared with the normal control group, PAAT of the model group showed significant decrease $(P<0.01)$; compared with the model group, STE high-, medium- and low-dose groups were significantly increased $(P<0.01)$. After 21 days of modeling, $75 \%$ animals in the model group had tricuspid regurgitation, while no occurrence of that in the STE treatment groups (Figure 1).

\section{Preventive Effect of STE in MCT-Induced PAH}

After 3 weeks of modeling, compared with the normal control group, mPAP, mRVP and max RVP of the model group were significantly increased $(P<0.01)$,

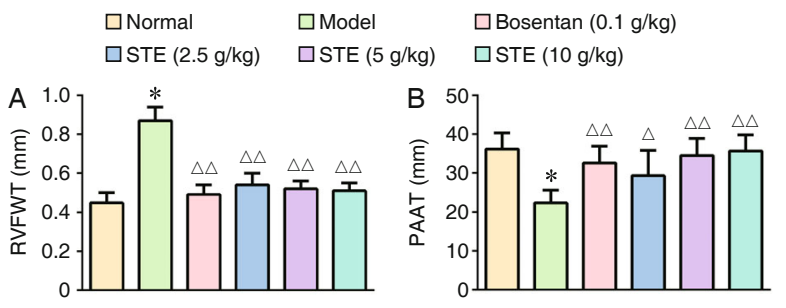

Figure 1. Effect of STE on RVFWT and PAAT in PAH Rats $(n=20, \bar{x} \pm s)$

Notes: ${ }^{*} P<0.01$, compared with the normal group; ${ }^{\triangle} P<0.05,{ }^{\triangle} P<0.01$; compared with the model group

and blood pressure (BP) was decreased $(P<0.05)$. Compared with the model group, mPAP, mRVP and max RVP of STE treated groups were significantly lower, and BP of STE-medium dose group was increased $(P<0.01$ or $P<0.05$, Figure 2$)$.

After modeling, compared with the normal control group, RV of the model group were significantly increased $(P<0.01)$. Compared with the model group, $\mathrm{RV}$ of STE treated groups were significantly lower $(P<0.01)$. Meanwhile, there was insignificant difference in $L V+S$ between groups $(P>0.05)$. However, STE had no significant effect on RVHI in PAH rats. After 3 weeks of modeling, compared with the normal control group, lungs wW and LI of the model group were significantly increased $(P<0.01)$. Compared with the model group, wW and LI of the STE treatment groups were significantly lower $(P<0.01$ or $P<0.05$, Figure 3$)$.

Compared with the model group, the STE treatment groups showed that thickness of pulmonary

Table 1. Comparison of Changes of Body Weight in Different Groups of Rats $(\bar{x} \pm s)$

\begin{tabular}{|c|c|c|c|c|c|}
\hline \multirow{2}{*}{ Group } & \multirow{2}{*}{$n$} & \multicolumn{4}{|c|}{ Body weight (g) } \\
\hline & & 1-day & 8-day & 15-day & 22-day \\
\hline Normal & 20 & $201.46 \pm 9.78$ & $238.11 \pm 11.34$ & $278.54 \pm 16.78$ & $318.82 \pm 25.10$ \\
\hline Model & 20 & $205.88 \pm 7.89$ & $237.36 \pm 10.56$ & $245.55 \pm 12.16$ & $254.30 \pm 10.54^{*}$ \\
\hline Bosentan & 20 & $206.21 \pm 9.63$ & $245.23 \pm 12.38$ & $279.02 \pm 17.46$ & $310.95 \pm 14.17^{\triangle}$ \\
\hline STE $(2.5 \mathrm{~g} / \mathrm{kg})$ & 20 & $204.62 \pm 10.81$ & $240.66 \pm 16.22$ & $285.33 \pm 21.80$ & $322.03 \pm 15.45^{\triangle}$ \\
\hline STE $(5 \mathrm{~g} / \mathrm{kg})$ & 20 & $203.02 \pm 10.30$ & $239.40 \pm 13.55$ & $281.29 \pm 9.10$ & $317.12 \pm 15.81^{\Delta}$ \\
\hline STE $(10 \mathrm{~g} / \mathrm{kg})$ & 20 & $200.66 \pm 10.88$ & $235.42 \pm 10.56$ & $279.62 \pm 23.22$ & $315.33 \pm 21.18^{\triangle}$ \\
\hline
\end{tabular}

Notes: ${ }^{*} P<0.05$, compared with the normal group; ${ }^{\triangle} P<0.01$, compared with the model group

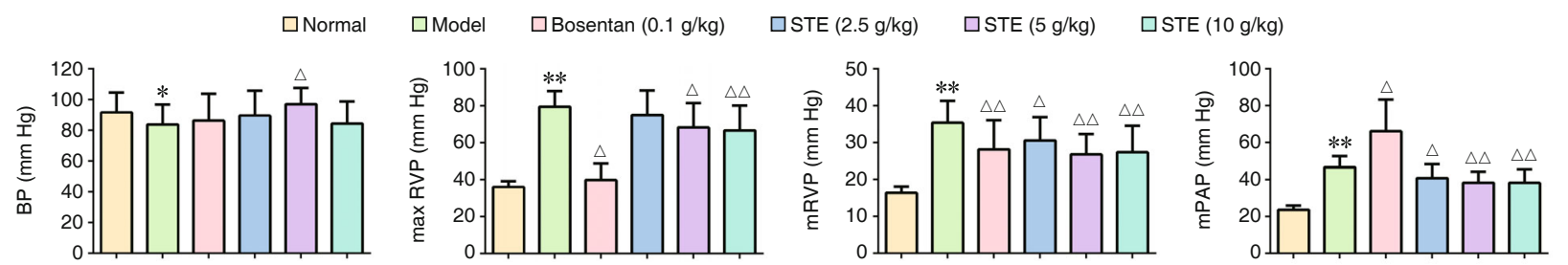

Figure 2. Effect of STE on BP, mPAP, mRVP and max RVP in PAH Rats $(n=20, \bar{x} \pm s)$ Notes: ${ }^{*} P<0.05,{ }^{* *} P<0.01$, compared with the normal group; ${ }^{\wedge} P<0.05,{ }^{\Delta} P<0.01$; compared with the model group 


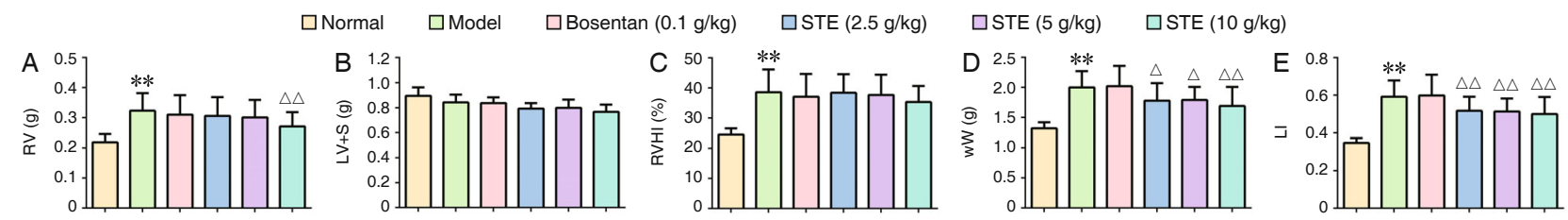

Figure 3. Effect of STE on Weight of Right Ventricle (RV), Left Ventricular Plus Septum (LV+S), Right Ventricular Hypertrophy Index (RVHI), Lungs Wet Weight (wW) and Lung Index (LI) in PAH Rats $(n=20, \bar{x} \pm s)$ Notes: ${ }^{*} P<0.05,{ }^{* *} P<0.01$, compared with the normal group; ${ }^{\Delta} P<0.05,{ }^{\triangle \Delta} P<0.01$; compared with the model group

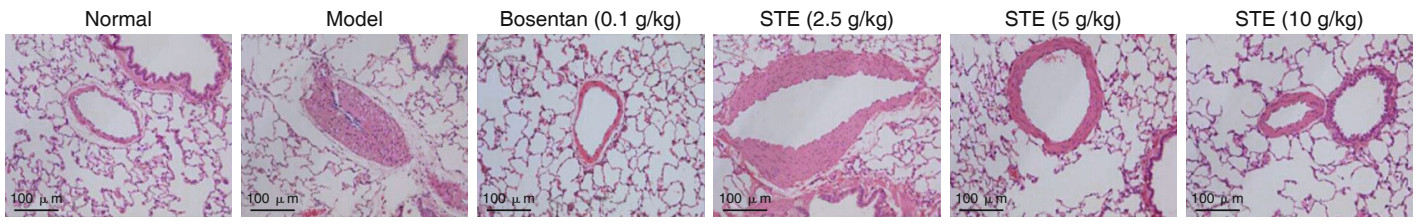

Figure 4. Effect of STE on Lung Inflammation of PAH Rats ( $\times 200$, HE staining)

arterioles decreased and lung inflammation significantly reduced (Figure 4).

Compared with the normal control group rats, the expression of $\alpha$-SMA in the small pulmonary artery of PAH model group rats was significantly increased. Compared with the model group rats, STE high-, medium-, and low-dose groups showed that the thickness of small pulmonary artery had a decreasing trend, and the expression of $\alpha$-SMA was significantly decreased. Compared with the normal control group rats, the expression of NF- $\kappa$ B p65 in the small pulmonary artery of PAH model group rats was significantly increased. Compared with the model group rats, STE group showed that the expression of NF- $\kappa$ B p65 was significantly decreased (Figure 5).
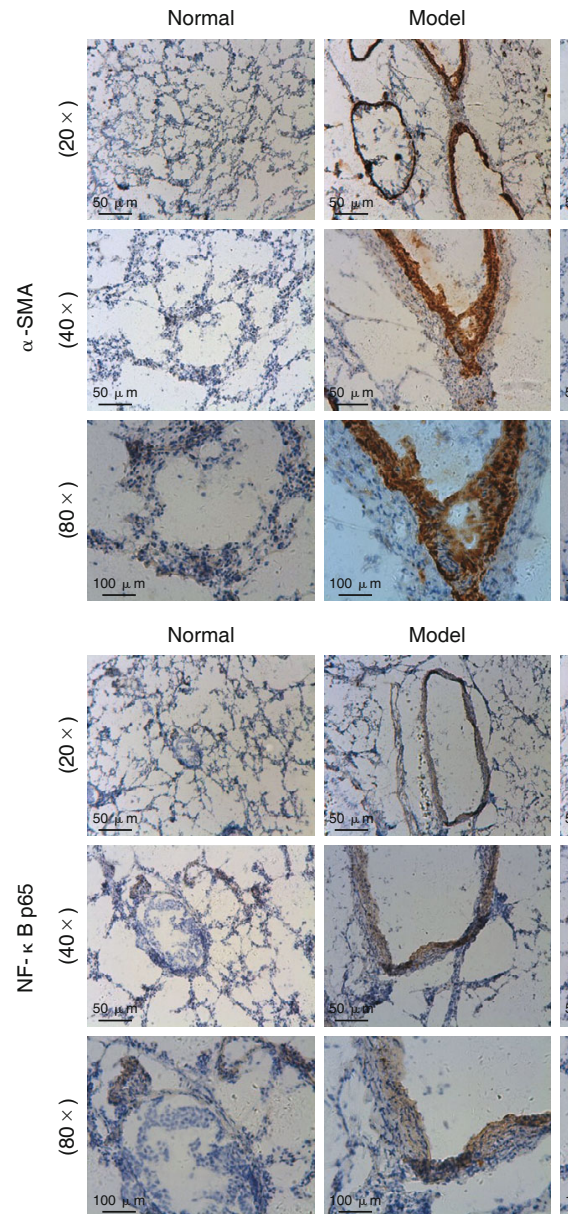

Bosentan $(0.1 \mathrm{~g} / \mathrm{kg})$

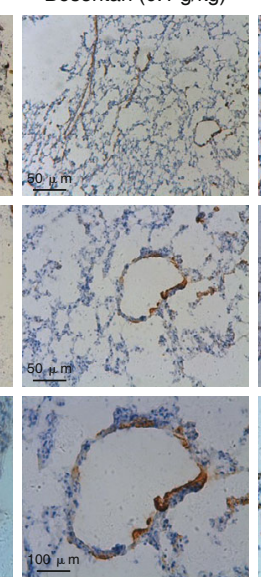

Bosentan $(0.1 \mathrm{~g} / \mathrm{kg})$
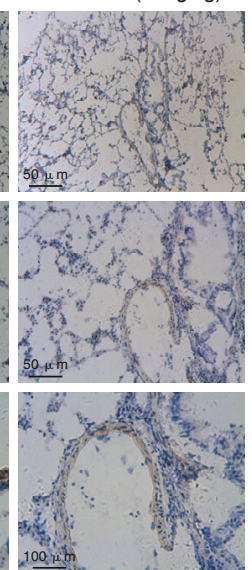

STE $(2.5 \mathrm{~g} / \mathrm{kg})$
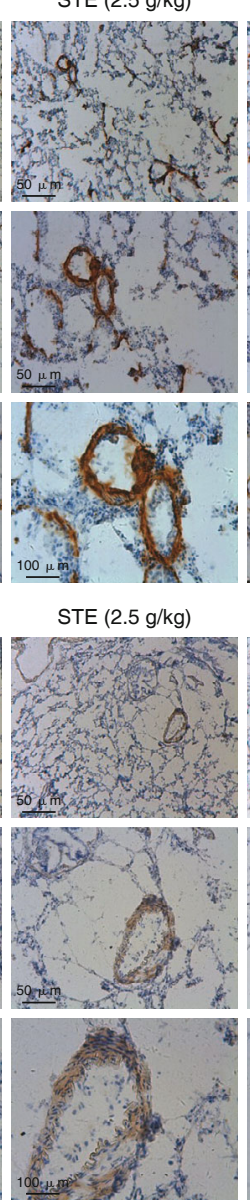

STE $(5 \mathrm{~g} / \mathrm{kg})$

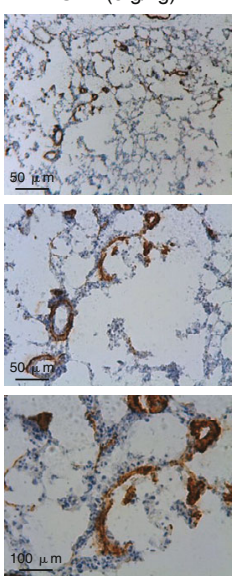

STE $(5 \mathrm{~g} / \mathrm{kg})$

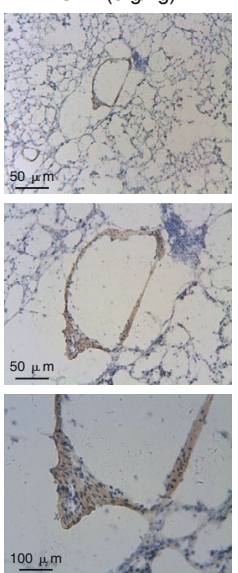

STE $(10 \mathrm{~g} / \mathrm{kg})$

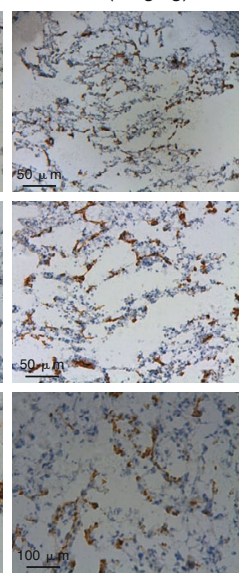

STE (10 g/kg)

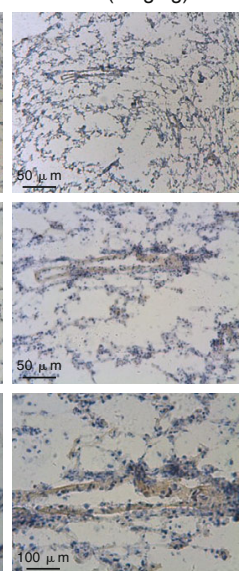

Figure 5. Effect of STE on $\alpha$-SMA and NF- $\kappa$ B p65 Expression of Lung Tissues in PAH Rats (HE staining) 


\section{DISCUSSION}

As a potentially lethal chronic disease, $\mathrm{PAH}$ presents with an increased right ventricular afterload and decreased endurance, and may lead to right heart failure and even death. The main therapies for PAH include oxygen therapy, maintenance of cardiac output, and use of anticoagulant and vasodilators. However, PAH remains incurable. In this study, our team early adopted different solvent to extract and separate for Scepteridium ternatum. Finally, petroleum ether extract $(1.53 \%)$, $86.7 \mathrm{~g}$ chloroform extract $(0.57 \%), 70 \mathrm{~g}$ ethyl acetate extract $(0.46 \%)$ and $293.5 \mathrm{~g} \mathrm{n}$-butanol extract $(1.94 \%)$ were obtained.

The above 4 extracts of Scepteridium ternatum were administered and compared with respect to their treatment effect in PAH. It was found that STE was the most effective extract. Therefore, in this study, STE was further investigated. This study showed that STE can relieve $\mathrm{PAH}$ by promoting pulmonary vascular remodelling and mitigating pulmonary inflammation. The rats in the model group showed signs of right heart failure, with an obvious increase in RV and wW. Rats in the STE high-dose group had a considerable reduction in $\mathrm{RV}$, wW and $\mathrm{LI}$ as compared with the model group $(P<0.01)$, thus indicating the treatment effect of STE on right heart failure induced by $\mathrm{PAH}$. mRVP, mPAP, RVHI, LI and pathological changes were measured as pharmacodynamic indicators of STE in PAH. According to the results, STE had a certain treatment effect on MCT-induced $\mathrm{PAH}$ in rats.

Abnormal proliferation of pulmonary arterial smooth muscle cells (PASMCs) can lead to pulmonary artery stenosis and increase pulmonary vascular resistance, which are important reasons underlying PAH pathogenesis. ${ }^{(9)}$ Hence inhibiting and reversing the proliferation of PASMCs and improving pulmonary arterial remodeling are important preventive measures. $\alpha$-SMA is an important marker of the smooth muscle cells, which reflects the contractility and proliferation of SMCs. ${ }^{(10)}$ It is found that $\alpha$-SMA is up-regulated in portal hypertension, idiopathic pulmonary fibrosis, radiation-induced lung injury and non-small cell lung cancer. The expression level is positively correlated with the severity of pathological lesions and therefore can be used as a diagnostic indicator. ${ }^{(11)}$ According to the latest research, overexpression of $\alpha$-SMA is closely related to abnormal proliferation of PASMCs, pulmonary artery remodeling and $\mathrm{PAH} .{ }^{(12)}$

In HE staining, pulmonary vascular thickness was obviously increased in the model group as compared with the normal control group. According to immunohistochemistry result, $\alpha$-SMA antigen was significantly up-regulated in the model group, indicating the presence of pulmonary vascular remodeling. For all STE treatment groups, the pulmonary arteriole thickness decreased with a down-regulation of $\alpha$-SMA, which suggested the role of STE in alleviating MCT-induced pulmonary vascular remodeling. Thus STE can inhibit $\alpha$-SMA expression, suppress inflammation and improve microcirculation and vascular diseases.

NF- $\kappa \mathrm{B}$ is a complex of $\mathrm{p} 6$ and $\mathrm{p} 50$ in Rel protein family, which is mainly in the form of p65 subunit in the lung tissue. ${ }^{(13)}$ In the cytoplasm of resting cells, NF- $\kappa \mathrm{B}$ and its inhibitor bound together, with no bioactivity, subjected to a variety of cytokines, virus, lipopolysaccharide stimulation in cells. Then I $\mathrm{B}$ was degraded, resulting in the separation of $I \kappa B$ and NF- $\kappa$ B and making NF- $\kappa B$ into the nucleus and DNA on the $\mathrm{KB}$ site binding to activate target gene transcription. ${ }^{(14)} \mathrm{At}$ present, it is believed that the NF- $\kappa$ B is a multifunctional nuclear transcription factor, with a wide range of biological activity and activation which could promote chemotaxis of cytokine, adhesion factor and procedures such as gene transcription. It plays an important role in the inflammatory response, and has close relationship with bronchial asthma, acute respiratory distress syndrome and pulmonary inflammatory diseases. ${ }^{(15)}$ Muraoka, et $\mathrm{al}^{(16)}$ reported that hypoxia can stimulate NF- $\kappa \mathrm{B}$ activation after receptor. Ao, et al ${ }^{(17)}$ reported that hypoxia can activate NF- $\kappa \mathrm{B}$ of pulmonary vascular smooth muscle cells, and its activation can inhibit the apoptosis of pulmonary vascular smooth muscle cell, promote its proliferation and eventually lead to pulmonary vascular remodeling. NF- $\kappa$ B plays an important role in the transcription of ET-1 gene, which can increase the production of ET-1 in vascular endothelial cells and lead to vascular contraction. ${ }^{(18)}$ Cheng, et $\mathrm{al}^{(19)}$ found the increased expression of NF- $\kappa$ B in lung tissue of hypoxic pulmonary hypertension rats. NF- $\kappa$ B mRNA expression was also increased in lung tissue of rats induced by MCT. ${ }^{(20)}$ Therefore, it can be concluded that NF- $\kappa$ B may play an important role in the inflammatory mechanism of PAH.

Compared with the normal control group, 
NF- $к$ B p65 expression was increased in the nuclei in the model group, but decreased in the STE treatment groups. This indicated the role of STE in inhibiting the MCT-induced entry of NF- $\kappa$ B into the nucleus. Thus the mechanism of STE is related to the suppression of NF- $\kappa$ B p65 expression, which further leads to the down-regulation of different inflammatory mediators, enzymes and receptors on the transcriptional level.

We have demonstrated through experiment that the STE relieved MCT-induced $\mathrm{PAH}$ in rats. STE may relieve pulmonary vascular disease and pulmonary injury by down-regulating the expression of NF- $\kappa$ B p65 and $\alpha$-SMA. However, the Chinese medicinal herbs usually contain a variety of active components and they work through multiple pathways and multiple targets, which is one of the reasons that the dose-effect relationship cannot be properly controlled. Further studies are required to identify the active components in STE and to determine the dose range, time-to-effect and other pathways for the treatment of $\mathrm{PAH}$.

\section{Conflict of Interest}

The authors declare no financial or commercial conflicts of interest.

\section{Author Contributions}

Huang $P, X$ in $W X$ and Fang $L$ designed the research. Zhong LK and Zheng XW performed research; Xin WX analyzed data; and Xin WX, Li QL and Huang P wrote the paper.

Electronic Supplementary Material: Supplementary material (Appendix) is available in the online version of this article at https://doi.org/10.1007/s11655-018-2573-6.

\section{REFERENCES}

1. Jardin F, Vieillard BA. Acute cor pulmonale. Cur Opin Crit Care 2009;15:67-70.

2. Yang SY, Luo XH, Feng EZ, Qi YS, Liu RN, Yin H, et al. Analysis of prognostic risk factors in the patients with acute exacerbation of chronic cor pulmonale and obstructive sleep apnea-hypopnea syndrome in high altitude area. Chin Crit Care Med (Chin) 2010;22:271-274.

3. Kjaergaard J, Akkan D, Iversen KK. Prognostic importance of pulmonary hypertension in patients with heart failure. Am J Cardiol 2007;99:1146-1150.

4. Lu HM, Liang XL, Zhang C, Liu Y. Effect of combination of sildenafil and beraprost or atorvastin on pulmonary arterial hypertension. Chin J Mod Appl Pharm (Chin) 2012;29:1134-1136.

5. Ying Y, Huang P, Yang B. Experimental research progress of traditional Chinese medicine treatment of pulmonary heart disease. Zhejiang J Integr Tradit West Med (Chin) 2013;23:75-78.

6. Ruan JS. Recent progress on research of Sceptridium ternatum and components. J China Pharm Univ (Chin) 2002;33(Suppl):328-329.

7. Zhuan J, Ruan JS. Study on the effect of Sceptridium ternatum pill on immune function of mice. Fujian $\mathrm{J}$ Tradit Chin Med (Chin) 2007;38(3):40-41.

8. Wang SM, Ruan JS. Inhibition of Sceptridium ternatum on mouseskin papilloma. Chin Med Mat (Chin) 2008;31:418-420.

9. Archer S, Rich S. Primary pulmonary hypertension: a vascular biology and translational research "work in progress". Circulation 2000;102:2781-2791.

10. Latourelle J, Fabry B, Fredberg JJ. Dynamic equilibration of airway smooth muscle contraction during physiological loading. J Appl Physiol 2002;92:771.

11. Xiao G, Luo DY, Liu YH. Non small cell lung cancer quality into fiber cells of a-SMA expression and significance. Jiangxi Pharm (Chin) 2010;45:207-209.

12. Dan LY, Li SH, Zhang HB. Monocrotaline on rat pulmonary artery a-SMA antigen had low expression and factor VIII antigen expression. Herald Med (Chin) 2010;29:415.

13. DiStefano A, Caramori G, Oates T. Inereased expression of nuclear factor-kappa $B$ in bronchial biopsies from smokers and palienis with COPD. Eur 2003,361(9368):1533-1544.

14. Blackwell TS, Christman JW. The role of nuclear factorkappa B in cytokine gene regulation. Am J Respir Cell Mol Biol 1997;17:3-9.

15. Christman JW, Sadikot RT, Blackwell TS. The role of nuclear factor-kappa $B$ in pulmonary diseases. Chest 2000;117:1482-1487.

16. Muraoka K, Shimizu K, Sun X. Hypoxia, but not reoxygenation, induces interleukin 6 gen-expression through NF-kappa B activation. Transplantation 1997;63:466-470.

17. Ao QL, Xiong M, Hao CR. Hypoxia of NF- $\kappa$ B and p53 in rat pulmonary artery smooth muscle cell proliferation and apoptosis change. J Huazhong Univ Sci Tech (Med Sci, Chin) 2002;31:4-6.

18. Ohkita M, Takaoka M, Sugii M, Shiota $Y$, Nojiri R. The role of nuclear factor-kappa $B$ in the regulation of endothelin-1 production by nitric oxide. Eur J Pharmacol 2003;472:159-164.

19. Cheng DY, Guan J, Xia XQ, Zhang Y, Su QL. The changes of nuclear factor kappa $B$ expression in lung tissue of hypoxic pulmonary hypertension rats. J Sichuan Univ (Med Sc, Chin) 2006;37:611-613.

20. Iwasaki T, Takahashi T, Shimizu H, Ohmori E, Morimoto T. Increased pulmonary heme oxygenase-1 and deltaaminolevulinate synthase expression in monocrotaline-induced pulmonary hypertension. Curr Neurovasc Res 2005;2:133-139. (Accepted February 24, 2017; First Online December 28, 2018) Edited by ZHANG Wen 